\title{
Pressure reconstruction of a planar turbulent flow field within a multiply-connected domain with arbitrary boundary shapes
}

\author{
X.Liu ${ }^{1 *}$, J. R. Moreto ${ }^{1}$ \\ ${ }^{1}$ San Diego State University, Department of Aerospace Engineering, San Diego, California, USA \\ *Xiaofeng.Liu@sdsu.edu
}

Abstract

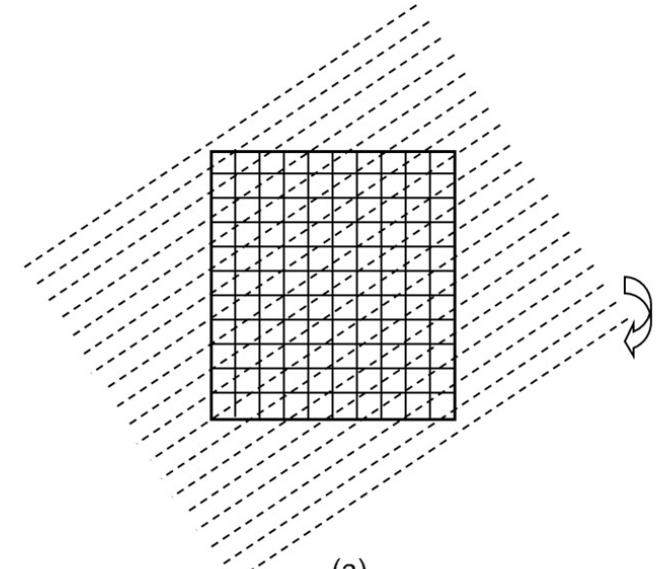

(a)

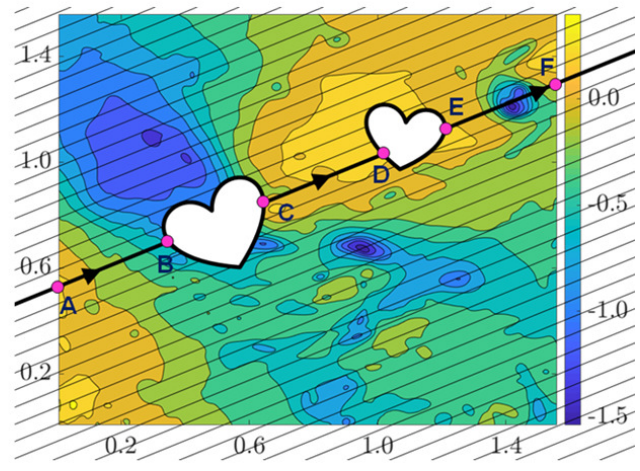

(b)

Figure 1: (a) The original rotating parallel ray omni-directional integration method for a simply-connected domain; (b) Illustration of implementation of the rotating parallel ray omni-directional integration algorithm in a multiply-connected domain.

Over the past two decades, it has been demonstrated that the instantaneous spatial pressure distribution in a turbulent flow field can be reconstructed from the pressure gradient field non-intrusively measured by Particle Image Velocimetry (PIV). Representative pressure reconstruction methods include the omnidirectional integration (Liu and Katz, 2006; Liu et al., 2016; Liu and Moreto, 2020), the Poisson equation approach (Violato et al., 2011; De Kat and Van Oudheusden, 2012), the least-square method (Jeon et al., 2015), and most recently, the adjoint-based sequential data assimilation method, which also essentially utilizes the Poisson equation to reconstruct the pressure(He et al., 2020). Most of these previous pressure reconstruction examples, however, were applied to simply-connected domains (Gluzman et al., 2017) only. None of these previous studies have discussed how to apply the pressure reconstruction procedures to a multiply-connected domain (Gluzman et al., 2017). To fill in this gap, this paper presents a detailed report for the first time documenting the implementation procedures and validation results for pressure reconstruction of a planar turbulent flow field within a multiply-connected domain that has arbitrary inner and outer boundary shapes. The pressure reconstruction algorithm used in the current study is the rotating parallelray omni-directional integration algorithm, which, as demonstrated in reference (Liu and Moreto, 2020) based on simply-connected flow domains, offers high-level of accuracy in the reconstructed pressure. While preserving the nature and advantage of the parallel ray omni-directional pressure reconstruction at places with flow data, the new implementation of the algorithm is capable of processing an arbitrary number of inner void areas with arbitrary boundary shapes. Validation of the multiply-connected domain pressure reconstruction code is conducted using the DNS (Direct Numerical Simulation) isotropic turbulence field 
available at the Johns Hopkins Turbulence Databases, with 1000 statistically independent pressure gradient field realizations embedded with random noise used to gauge the code performance. For further validation, the code is also applied for pressure reconstruction from the DNS pressure gradient in the ambient flow field of a shock-induced non-spherical bubble collapse in water (Johnsen and Colonius, 2009). The successful implementation of the parallel ray pressure reconstruction method to multiply-connected domains paves the way for a variety of important applications including, for example, experimental characterization of pressure field changes during the process of cavitation bubble inception, growth and collapse, non-intrusive unsteady aerodynamic force assessment for an arbitrary body shape immersed in flows, and multi-phase flow investigations, etc. In particular, as an immediate follow-up effort, the parallel ray pressure code will be used for the instantaneous pressure distribution reconstruction of the turbulent flow surrounding cavitation inception bubbles occurring on top of a cavity trailing corner based on high-speed PIV measurements.

The original rotating parallel ray omni-directional integration method for simply-connected domain is illustrated in Figure 1 $(\mathrm{a})$. This method utilizes parallel rays as guidance paths for line integration of pressure gradient. By rotating the parallel rays, effectively omni-directional paths with equal weights coming from all directions toward the point of interest at any location within the computation domain can be generated. To reduce the effect of erroneous pressure along the boundary, the parallel ray omni-directional integration algorithm utilizes iterations of boundary pressure values in the integration process. Initially, integration along the perimeter of the measurement domain gives the initial boundary pressure values, but since each boundary node is crossed by numerous paths, the initial boundary values can be corrected/replaced with the results of the omni-directional integration. The integration process is then repeated using the new boundary values and the iterations proceed until the results converge within an acceptable threshold level, thus achieving accurate Dirichlet conditions for boundary pressure values. The convergence of the boundary pressure calculation follows an exponential decay fashion, as demonstrated theoretically in Liu and Moreto (2020). Once the accurate boundary pressure values are achieved, the pressure values over the entire domain can then be determined by invoking the final round of the omni-directional integration across the domain.

The aforementioned key features of the rotating parallel ray omni-directional integration are preserved during the implementation of the same algorithm in a multiply-connected domain with arbitrary domain boundary shapes, as shown in Figure 1(b). A bold parallel ray serving as guidance for the line integration penetrates the planar domain at boundary point A. Along the way it encounters the inner void boundaries at points B, C, D and E, respectively, and finally leaves the entire domain at boundary point F. Pressure values at all the boundary points identified in this way, including both the inner and the outer domain boundary points, will then go through the boundary value iteration process, as outlined in the procedures described in Liu and Moreto (2020). After achieving convergence, the pressure at inner nodal points within the multiplyconnected domain will then be calculated. Please note during the entire pressure reconstruction process, nodal points within the void areas are not involved in any form of calculations.

Sample results for pressure reconstruction within multiply-connected domains based on the Hopkins isotropic turbulence database are shown in Figure 2. Performance assessment based on the 1000 statistically independent pressure gradient field realizations with the added random noise (will show in the presentation) indicates that, like the simply-connected domain case demonstrated in Liu and Moreto (2020), the parallel ray algorithm outperforms the Poisson equation approach in terms of the reconstructed pressure accuracy. As an example of further validation, Figure 3 shows one sample comparison of the instantaneous DNS pressure and the corresponding reconstructed pressure using the parallel ray code. The 2- $\sigma$ deviation of the pressure difference between the reconstructed and the original DNS pressures shown in the Figure 3(d) is $0.03 \%$ of the maximum pressure (i.e., the shock pressure $p_{s}$ ) in the field. Please note that the procedures described in this paper can be readily extended to 3D applications.

\section{Acknowledgements}

This work has been sponsored by the Office of Naval Research Grant No. N00014-21-1-2392 (Dr. K.-H. Kim is the Program Officer). The authors would like to thank Professor Tim Colonius for his permission of the use of his shock-induced non-spherical bubble collapse DNS data in this research. The help from Mr. Jose Rodolfo Chreim in retrieving the bubble collapse DNS data, and the help from Dr. Minping Wan and Professor Charles Meneveau in retrieving the isotropic turbulence DNS data from JHTDB are all gratefully acknowledged. Both authors contributed equally to this paper. 

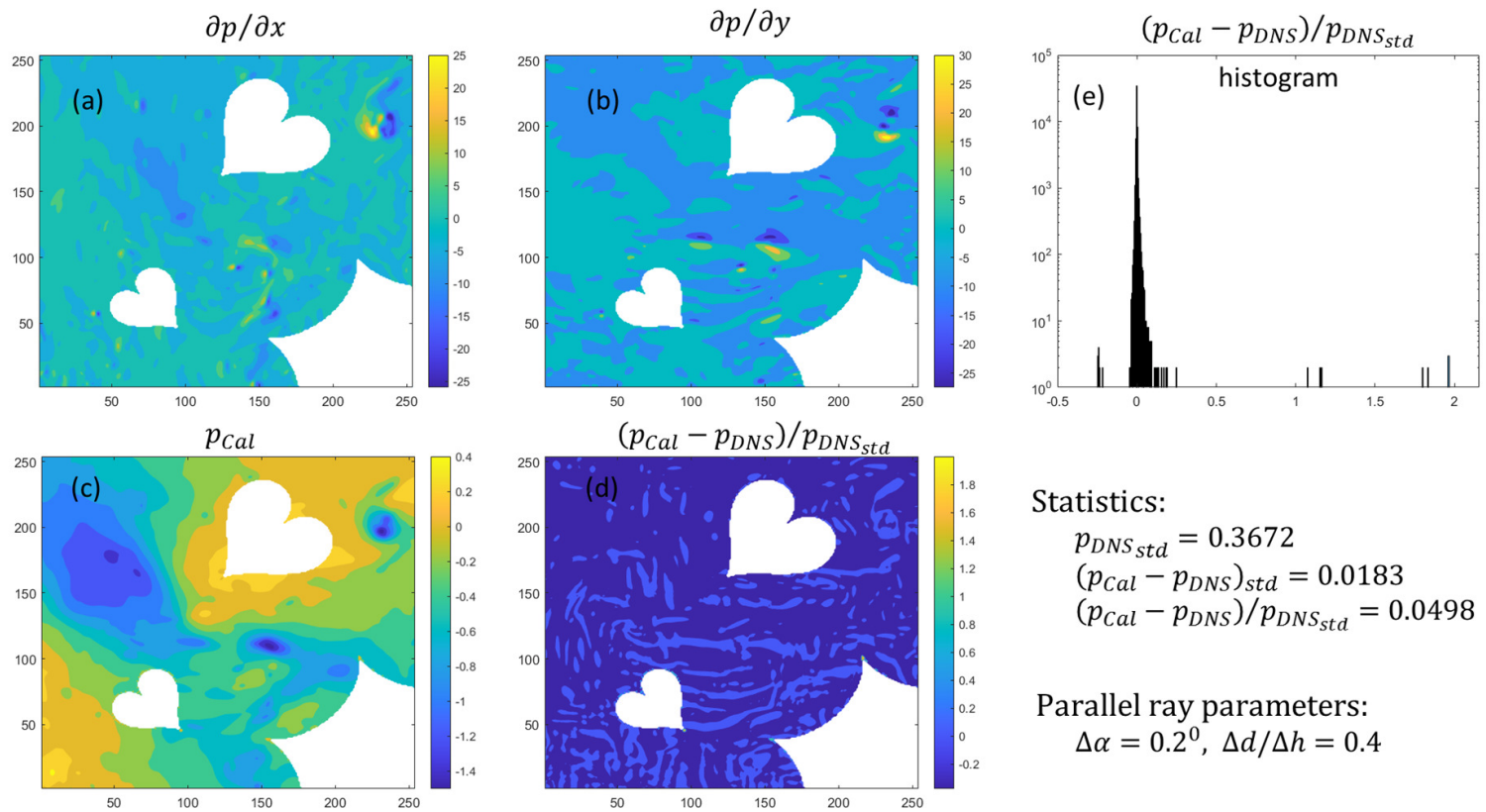
Statistics:
$p_{D N S_{S t d}}=0.3672$
$\left(p_{C a l}-p_{D N S}\right)_{s t d}=0.0183$
$\left(p_{\text {Cal }}-p_{D N S}\right) / p_{D N S_{\text {std }}}=0.0498$

Parallel ray parameters:

$\Delta \alpha=0.2^{0}, \Delta d / \Delta h=0.4$

Figure 2: Sample pressure reconstruction results using the parallel ray code for a multiply-connected domain with arbitrary boundary shapes based on a DNS isotropic turbulence database. (a) and (b), pressure gradient distributions; (c) reconstructed pressure distribution; (d) difference between the reconstructed and the original DNS pressure fields; (e) histogram of the difference between the reconstructed and the DNS pressure fields.

\section{References}

De Kat R and Van Oudheusden B (2012) Instantaneous planar pressure determination from piv in turbulent flow. Experiments in fluids 52:1089-1106

Gluzman S, Mityushev V, and Nawalaniec W (2017) Computational analysis of structured Media. Academic Press

He C, Liu Y, and Gan L (2020) Instantaneous pressure determination from unsteady velocity fields using adjoint-based sequential data assimilation. Physics of Fluids 32:035101

Jeon YJ, Chatellier L, Beaudoin A, and David L (2015) Least-square reconstruction of instantaneous pressure field around a body based on a directly acquired material acceleration in time-resolved piv. in Symp. part. image velocim.-PIV15

Johnsen E and Colonius T (2009) Numerical simulations of non-spherical bubble collapse. Journal of fluid mechanics 629:231-262

Liu X and Katz J (2006) Instantaneous pressure and material acceleration measurements using a fourexposure piv system. Experiments in fluids 41:227-240

Liu X and Moreto JR (2020) Error propagation from the piv-based pressure gradient to the integrated pressure by the omnidirectional integration method. Measurement Science and Technology 31:055301

Liu X, Moreto JR, and Siddle-Mitchell S (2016) Instantaneous pressure reconstruction from measured pressure gradient using rotating parallel ray method. in 54th AIAA Aerospace Sciences Meeting. page 1049

Violato D, Moore P, and Scarano F (2011) Lagrangian and eulerian pressure field evaluation of rod-airfoil flow from time-resolved tomographic piv. Experiments in fluids 50:1057-1070 

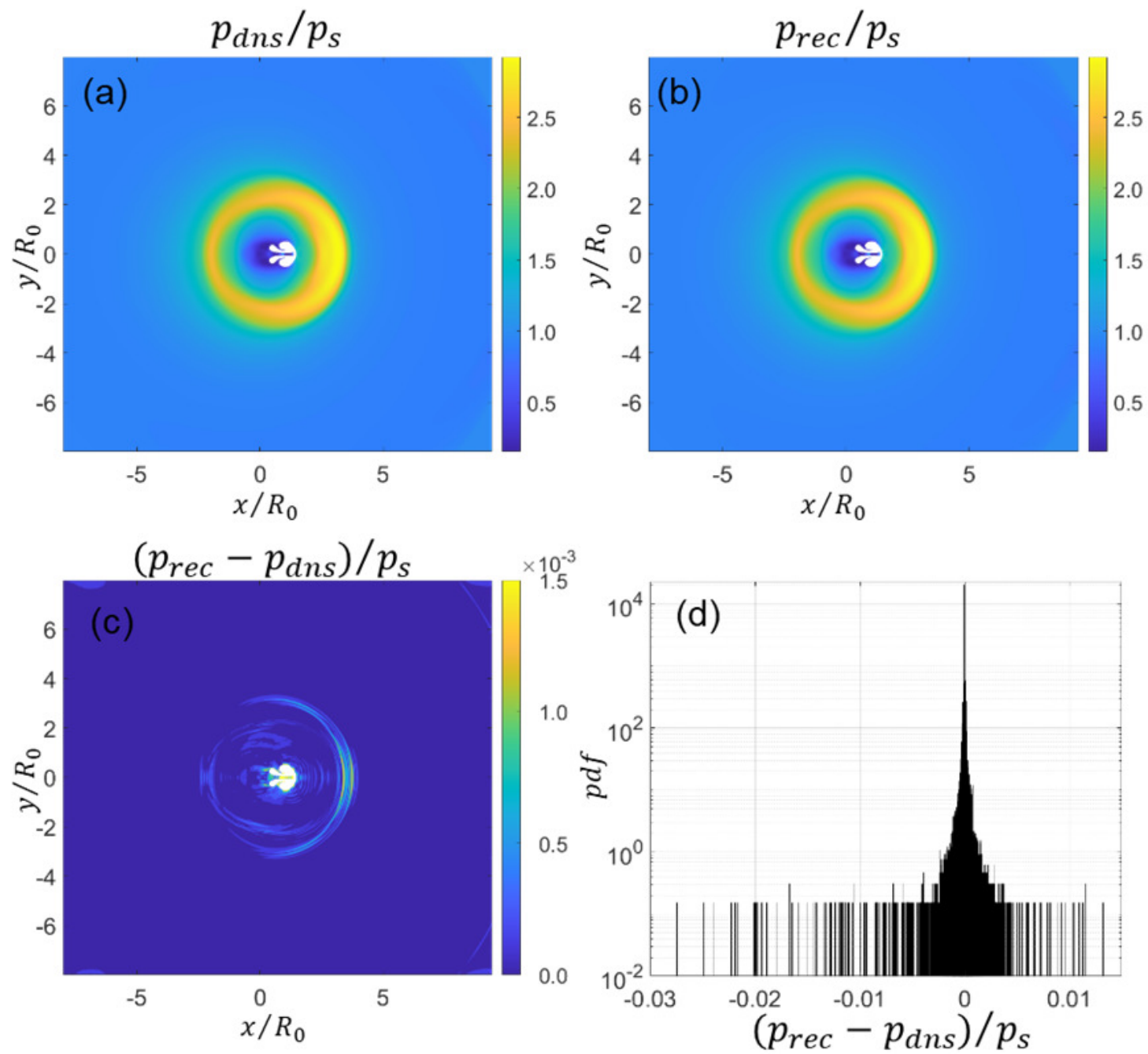

Figure 3: Sample instantaneous pressure reconstruction from the DNS pressure gradient of a shock-induced non-spherical bubble collapse in water. (a) The DNS pressure from Johnsen and Colonius (2009); (b) The reconstructed pressure; (c) The difference between the reconstructed and the original DNS pressure fields, normalized by the maximum pressure i.e., the shock pressure $p_{s}$; (d) the Probability Density Function (pdf) of the relative pressure difference. The white region in the plots indicates the deformed bubble during the collapse process. 